\title{
The implications from CANGAROO-III observations of TeV blazar PKS 2155-304
}

\author{
K. Nishijima, Y. Sakamoto, J. Kushida and K. Saito (on behalf of the \\ CANGAROO-III Collaboration)
}

\author{
Department of Physics, Tokai University, Hiratsuka, Kanagawa 259-1292, Japan
}

\begin{abstract}
We have observed the high-frequency-peaked BL Lacertae object PKS 2155-304 in 2004, 2005 and 2006 with the CANGAROO-III imaging atmospheric Cherenkov telescope, and have detected a signal above $660 \mathrm{GeV}$ at the $4.8 \sigma$ level during the 2006 outburst period. Intranight flux variability on time scale of half an hour is observed. From this variability time scale, the size of the TeV gamma-ray emission region is restricted to $5 \times 10^{13} \delta \mathrm{cm}$, and the super massive black hole mass is estimated to be less than $1.9 \times 10^{8} \delta \mathrm{M}_{\text {Solar }}$, where $\delta$ is the beaming factor. The differential energy spectrum is obtained, and an upper limit of the extragalactic infrared background light (EBL) flux is derived under some assumption. We also fit a synchrotron self Compton (SSC) model to the spectral energy distribution (SED) and derive the beaming factor and magnetic field strength.
\end{abstract}

Keywords: Blazars, PKS 2155-304

PACS: $95.85 . \mathrm{Pw}, 98.54 . \mathrm{Cm}$

\section{INTRODUCTION}

The first detection of $\mathrm{TeV}$ gamma rays from PKS 2155-304 was reported by the Durham group during the active phase in $1997[1]$. Although the CANGAROO-I and the CANGAROO-II observations in 1997, 1999, 2000, and 2001 could not fi nd an excess signal from PKS 2155-304 [2, 3, 4, 5], it was confi rmed by the H.E.S.S. group as a TeV gamma-ray source in 2004 [6]. In July 2006, the extreme TeV fhre was detected by the H.E.S.S. group, and they reported very beautiful light curve with 1 minute timescale resolution [7]. Some modeling for the rapid TeV fux variability and the spectral energy distribution of PKS 2155-304 are reported $[8,9,10,11]$, and intensive interpretations of the data have been attempted.

CANGAROO-III observations of PKS 2155-304 have been performed from 2004 to 2006. Particularly in 2006, target-of-opportunity observations were made triggered by the H.E.S.S. report. In this paper, we summarize our observations and the results particularly focused on the data of the 2006 outburst period [12], and give some implications from them.

\section{OBSERVATIONS AND ANALYSIS}

The CANGAROO-III imaging atmospheric Cherenkov telescope system is operated in Woomera, South Australia (longitude $136^{\circ} 47^{\prime} \mathrm{E}$, latitude $31^{\circ} 06^{\prime} \mathrm{S}, 160 \mathrm{~m}$ a.s.1.). Three of the four telescopes were used in these observations. The details of the CANGAROO-III telescope system are described in [13, 14, 15].

Observations of PKS 2155-304 in 2004 were made by local trigger using three telescopes. From 2005, an on-line stereo trigger system became available. The details of the CANGAROO-III trigger system are given in $[16,17]$. In 2005 , observations were made with only two telescopes due to electronics problems. Observations in 2006 consist mostly a three-telescope confi guration, but the exception to this is in a part of July due to a mechanical tracking problem with the third telescope. These observations were made using wobble mode, in which the pointing position of each telescope was shifted in declination by $\pm 0.5^{\circ}$ from the center of PKS 2155-304 alternatively every 20 minutes. Observation periods, observation nights, the amount of observation time, the number of telescopes available, and the trigger mode for each year are summarized in Table 1. Typical trigger rate of the two-fold and the three-fold coincidences on July 2006 observations were $\sim 20 \mathrm{~Hz}$ and $\sim 12 \mathrm{~Hz}$, respectively.

After the image cleaning and the reconstruction of the arrival direction, we applied the Fisher discriminant (FD) method to the data in order to reject numerous cosmicray background events[18]. Following a Monte Carlo simulation study, the optimum FD cut values were determined, and the $\theta^{2}$ cuts were applied, for example at $\theta^{2}<0.06 \mathrm{deg}^{2}$ for the three fold data. Details of the observations in 2006 and analysis method are described in [12], and references therein. After the data reduction described above, the effective live times are $17.0 \mathrm{hrs}$, $38.6 \mathrm{hrs}, 25.1 \mathrm{hrs}$, and $17.1 \mathrm{hrs}$ in 2004, 2005, July 2006, and August 2006, respectively. 
TABLE 1. Summary of CANGAROO-III observations of PKS 2155-304

\begin{tabular}{ccrccc}
\hline Year & Period & Nights & Obs. time [hrs] & $\sharp$ of tel. & Stereo mode \\
\hline 2004 & Aug.8-23 & 11 & 20.5 & 3 & off-line \\
2005 & June 6-15 & 6 & 46.8 & 2 & on-line \\
& July 1-5 & 5 & $\uparrow$ & 2 & on-line \\
& July 29-Aug.5 & 12 & $\uparrow$ & 2 & on-line \\
2006 & July 28-Aug.2 & 5 & 29.0 & $3 \& 2$ & on-line \\
& Aug.17-25 & 6 & 19.1 & 3 & on-line \\
\hline
\end{tabular}

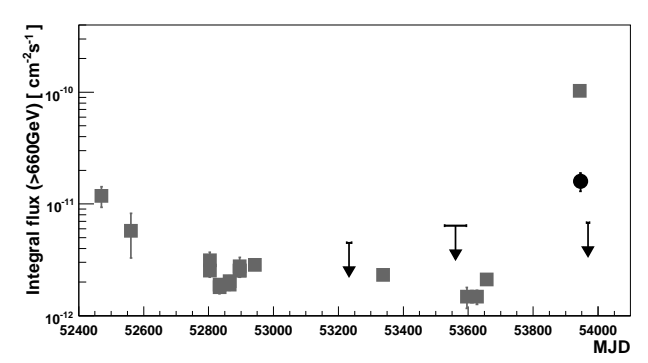

FIGURE 1. Long-term variations of the flux of PKS 2155304 (black closed circle and bars with an arrow) compared to H.E.S.S. results (gray squares)[7, 21]. The flux of the H.E.S.S. are converted to the values above $660 \mathrm{GeV}$ assuming a photon index of 3.3 .

\section{RESULTS}

In 2004 and 2005, no significant excess events were found and $2 \sigma$ upper limits on the integral flux were obtained: $4.5 \times 10^{-12} \mathrm{~cm}^{-2} \mathrm{~s}^{-1}$ above $580 \mathrm{GeV}$ for 2004 and $6.4 \times 10^{-12} \mathrm{~cm}^{-2} \mathrm{~s}^{-1}$ above $660 \mathrm{GeV}$ for 2005 . In July 2006, just after the largest outburst reported by the H.E.S.S. group[19], we detected $322 \pm 67$ excess events corresponding to $4.8 \sigma$ level from the direction of PKS 2155-304[12]. The time-averaged integral flux above $660 \mathrm{GeV}$ is calculated to be $F(>660 \mathrm{GeV})=$ $\left(1.6 \pm 0.3_{\text {stat }} \pm 0.5_{\text {syst }}\right) \times 10^{-11} \mathrm{~cm}^{-2} \mathrm{~s}^{-1}$, which corresponds to $\sim 45 \%$ of the flux observed from the Crab Nebula [20]. In the follow-up observations in August 2006, only the flux upper limit ( $2 \sigma$ level) was obtained: $F(>660 \mathrm{GeV})<6.4 \times 10^{-12} \mathrm{~cm}^{-2} \mathrm{~s}^{-1}$ (20\% Crab flux), which means the $\mathrm{TeV}$ gamma-ray activity subsided in August. These results are summarized in Table 2. Our results obtained with the CANGAROO-III telescope for each year are compared to the H.E.S.S. results in Fig. 1, where the fluxes by the H.E.S.S. are converted to the values above $660 \mathrm{GeV}$ assuming a photon index of 3.3 and plotted.

Fig. 2 and 3 show the nightly average integral flux above $660 \mathrm{GeV}$ in 2006. From the light curve, it is seen that the averaged flux reached $\sim 70 \%$ of Crab flux level on the night of July 30 . Assuming a constant average flux between July 28 and August 2, a $\chi^{2}$ fi t yields a value of

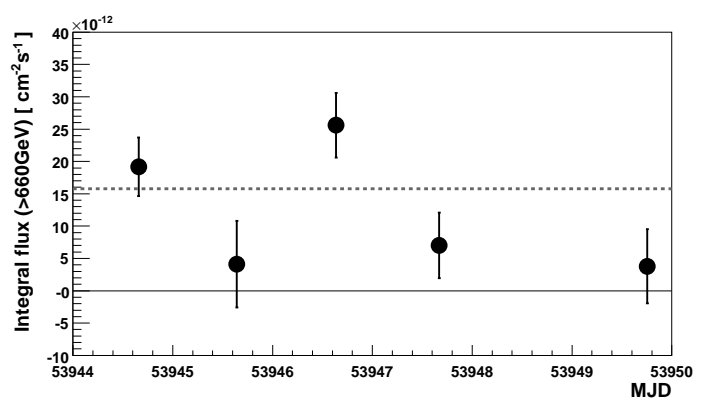

FIGURE 2. Daily light curve of PKS 2155-304 from July 28 to August 2 in 2006 expressed by the integral flux above $660 \mathrm{GeV}$. The dashed line indicates an average integral flux during this observation period.

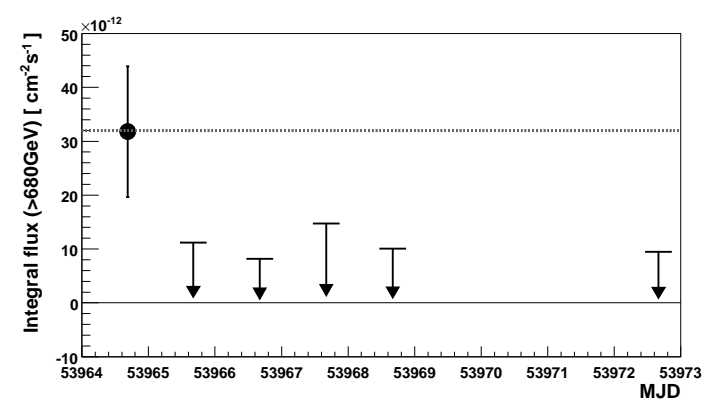

FIGURE 3. Daily light curve of PKS 2155-304 from August 17 to 25 in 2006 expressed by the integral flux above $660 \mathrm{GeV}$. The dotted line indicates one Crab flux level above $660 \mathrm{GeV}$.

13.9 for 4 degree of freedom, which corresponds to a $\chi^{2}$ probability of $\sim 8 \times 10^{-3}$.

We further investigate intranight variation by dividing the data into $\sim 40$ minute bins for each night in July 2006. The light curve is shown in Fig. 4. Assuming a constant average flux for each night, $\chi^{2}$ test for the data of July 28 and 30 gives $\chi^{2} /$ dof $=29.2 / 6$ and $22.1 / 6$, respectively, which correspond to $\chi^{2}$ probability of $6 \times 10^{-5}$ and $1 \times 10^{-3}$, respectively. The intranight variations are apparent. The fractional root mean square variability amplitudes $F_{\text {var }}$ are calculated to be $0.75 \pm 0.07$ on July 28 and $0.58 \pm 0.08$ on July 30 . These values are comparable to the variability reported by the 
TABLE 2. Summary of results for PKS2155-304, where 2006 July corresponds to period from July 28 to August 22006

\begin{tabular}{|c|c|c|c|c|c|c|}
\hline Year & & $\begin{array}{c}E_{t h} \\
{[\mathbf{G e V}]}\end{array}$ & $\begin{array}{l}\text { Excess } \\
\text { events }\end{array}$ & $\begin{array}{c}\text { Significance } \\
{[\sigma]}\end{array}$ & $\begin{array}{c}\text { Flux } \\
{\left[10^{-12} \mathbf{c m}^{-2} \mathbf{s}^{-1}\right]}\end{array}$ & $\begin{array}{c}\text { Crab flux } \\
{[\%]}\end{array}$ \\
\hline 2004 & & 580 & $-14 \pm 28$ & -0.5 & $<4.5$ & $<10$ \\
\hline 2005 & & 660 & $64 \pm 53$ & 1.2 & $<6.4$ & $<20$ \\
\hline \multirow[t]{2}{*}{2006} & July & 660 & $322 \pm 67$ & 4.8 & $16 \pm 3_{\text {stat }} \pm 5_{\text {syst }}$ & 45 \\
\hline & Aug. & 660 & $-1 \pm 30$ & -0.0 & $<6.5$ & $<20$ \\
\hline
\end{tabular}

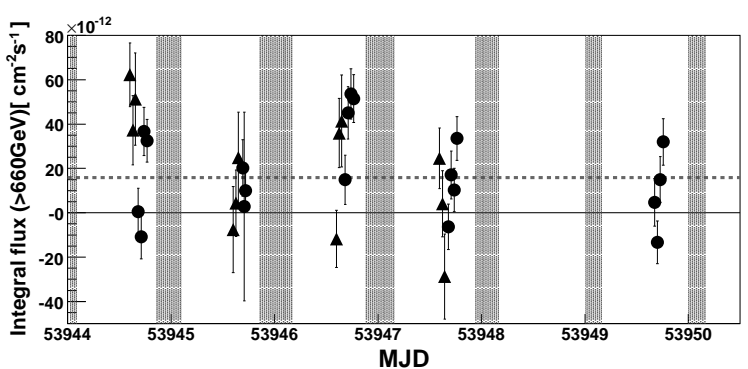

FIGURE 4. Light curve of PKS 2155-304 expressed by the integral flux above $660 \mathrm{GeV}$ between July 28 and August 2 based on a 40 minute bin width. Triangles and closed circles indicate the flux obtained from the two-fold and three-fold data set, respectively.The dashed line shows an average integral flux during this observation period. The shaded areas indicate the H.E.S.S. observation periods. The time difference between the H.E.S.S. and CANGAROO-III sites is $\sim 8$ hours.

H.E.S.S. [7]. Doubling times are also calculated from Fig. 4. We obtained the shortest doubling time of 34 minutes as an $1 \sigma$ upper limit.

A photon index of the time-averaged differential energy spectrum of PKS 2155-304, which was obtained between July 28 and August 2 in 2006, is $\Gamma=-2.5 \pm 0.5_{\text {stat }} \pm 0.7_{\text {syst }}$, and a flux normalization at $1 \mathrm{TeV}$ is $N(1 \mathrm{TeV})=\left(1.0 \pm 0.2 \pm_{\text {stat }} \pm_{\text {syst }}\right) \times$ $10^{-11} \mathrm{~cm}^{-2} \mathrm{~s}^{-1} \mathrm{TeV}^{-1}[12]$.

\section{DISCUSSION}

Assuming that the gamma-rays from PKS 2155-304 are generated in the jet which is directed toward us with a beaming factor $\delta$, the observed variability time scale $t_{v a r}$ is related to the size $R$ of emission region by the equation $R<\delta c t_{v a r} /(1+z)$ from causality, where $z$ is the redshift. Using an upper limit of the shortest doubling time as an variability time scale, i.e. $t_{\text {var }}=34$ minutes, the size of $\mathrm{TeV}$ gamma ray emission region is limited to $R<5 \times 10^{13} \delta \mathrm{cm}$. The central engine of a blazar is believed to contain a super massive black hole. If we assume that the size of emission region is larger than the Schwarzschild radius $R_{S c h}$, i.e. $R>R_{S c h}$, a black hole mass $M_{B}$ is less than $\left(c^{2} / 2 G\right) R$. Then, substituting numerical values of constant, an upper limit of a super massive black hole mass is estimated to be $M_{B}<1.9 \times$ $10^{8} \delta \mathrm{M}_{\text {Solar }}$, where $\mathrm{M}_{\text {Solar }}$ is one Solar mass. On the other hand, assuming an internal shock model in the jet, where the second blob catching up with the first blob and create a shock wave, we estimate the distance $D$ from the center of black hole to the shock region as $D=10^{3}(k / 10)(\gamma / 10)^{2}\left[2 \alpha^{2} /\left(\alpha^{2}-1\right)\right] R_{S c h}$. Here $\gamma$ and $\alpha \gamma$ are Lorentz factors of the first and the second blobs $(\alpha>1)$, respectively, and blobs are assumed to be emitted at a time interval of $\sim k R_{S c h} / c(k>3)$. The size of gamma-ray emission region is $D / \gamma$ and observed variability time scale is $t_{v a r} \sim D / c \gamma^{2}$. So the black hole mass is expressed as

$$
M=1.0 \times 10^{6}\left(\frac{t_{\text {var }}}{\sec }\right)\left(\frac{10}{k}\right)\left(\frac{\alpha^{2}-1}{2 \alpha}\right) \mathrm{M}_{\text {Solar }} .
$$

Substituting $t_{\text {var }}=34$ minutes, and assuming $\alpha \gg 1$ and $k>3$, the black hole mass is estimated to be less than $3.4 \times 10^{9} \mathrm{M}_{\text {Solar }}$.

As is well known, $\mathrm{TeV}$ photons are absorbed by an interaction with extragalactic infrared background light (EBL) through the pair production process $\gamma_{\mathrm{TeV}}+$ $\gamma_{E B L} \rightarrow e^{+}+e^{-}$. Measured flux $F_{m}$ is related to the source flux by $F_{m}=e^{-\tau} F_{s}$, where $\tau$ is the optical depth of the above process. Assuming an intrinsic photon index is not harder than -1.5[22] and no absorption below $200 \mathrm{GeV}$, the upper limit of the optical depth $\tau$ is calculated from the observed spectrum. Adopting the $1 \sigma$ upper limit of the observed spectrum in July 2006, the optical depth, for example at $1.1 \mu \mathrm{m}$, is calculated to $\tau=2.3$, and an upper limit on the EBL flux can be estimated to be $45.5 \mathrm{nWm}^{-2} \mathrm{sr}^{-1}$ for $1.1 \mu \mathrm{m}$.

On the contrary, assuming the EBL density model, an intrinsic spectrum can be estimated from the measured spectrum. Choosing the EBL density model of Primack et al.[23], an intrinsic spectrum of $d N / d E=(2.7 \pm$ $0.6) \times 10^{-11}(E / 1 \mathrm{TeV})^{-(1.8 \pm 0.6)} \mathrm{cm}^{-2} \mathrm{~s}^{-1} \mathrm{TeV}^{-1}$ is derived from the observed spectrum. When applying the model by Stecker et al.[24] to the observed spectrum, the deabsorbed spectrum seems to be too hard.

Using the intrinsic energy spectrum derived above, the simple SSC model by Kino et al.[25] is applied 


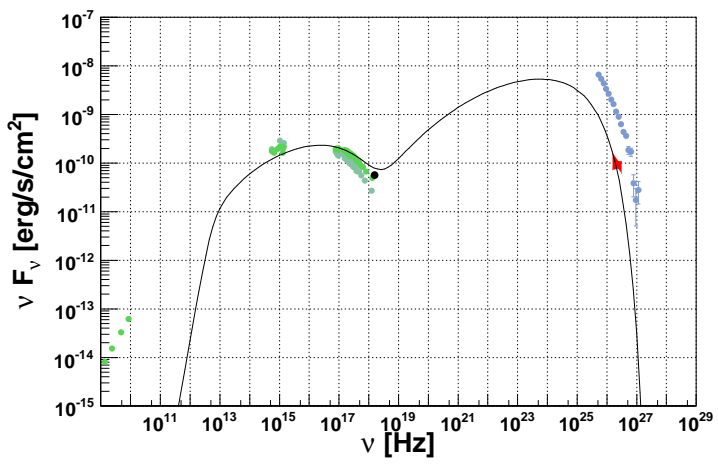

FIGURE 5. Spectral energy distribution of PKS 2155-304 in 2006. Red butterfly area indicates the deabsorbed average flux above $660 \mathrm{GeV}$ observed with the CANGAROO-III telescope between July 28 and August 2, and light blue shows the results by H.E.S.S. on July 27[7]. Green plots indicate the data of XTE and UVOT on Swift[8] and Narrabri on July 30. Deep green and black indicate Swift data on Aug.2 and RXTE data on July 30, respectively.

to the spectral energy distribution. Figure 5 shows the spectral energy distributions of multiwavelength observations. Adopting $5 \times 10^{13} \delta \mathrm{cm}$ as the radius of the emission region based on our results, fi tting was done to our average spectral energy distribution between July 28 and August 22006 and X-ray data taken by Swift on July 30 , which are not perfectly simultaneous observations. We found that the beaming factor $\delta \sim 60$ and magnetic fi eld $B \sim 2.5 \mathrm{G}$ explain the observed spectral energy distribution well. This beaming factor is much greater than the typical value for blazars and is consistent with values obtained by other $[8,10]$. However the magnetic fi eld is stronger compared to those they derived. We also try to fi $t$ the H.E.S.S. data and we found the smaller size of emission region and higher electron density are required, and beaming factor $\delta \sim 80$ and magnetic fi eld $B \sim 5.0 \mathrm{G}$ are obtained. However, it is still poorly-fi tting, and we need to consider more complicated and realistic model.

\section{CONCLUSION}

We observed the nearby HBL PKS 2155-304 in 2004, 2005, and 2006 with the CANGAROO-III imaging atmospheric Cerenkov telescope. During the active phase in July 2006, we detected a signal at $4.8 \sigma$ level $(\sim$ $45 \%$ Crab flux) above $660 \mathrm{GeV}$. The intranight time variations of the flux were observed, and obtained shortest doubling time was 34 minutes. The size of $\mathrm{TeV}$ gammaray emission region is limited to $5 \times 10^{13} \delta \mathrm{cm}$ from the variability time scale, and a super massive black hole mass is restricted to be less than $1.9 \times 10^{8} \delta \mathrm{M}_{\text {Solar }}$, where $\delta$ is a beaming factor. From the differential energy spectrum, an upper limit of the extragalactic infrared background light (EBL) flux is derived under the model assumption. The synchrotron self Compton (SSC) model fi tting suggests a larger beaming factor compared to typical blazars and a rather larger magnetic strength.

\section{ACKNOWLEDGMENTS}

We thank Dr. W. Hofmann, Dr. S. Wagner, Dr. G. Rowell, Dr. W. Benbow, Dr. B. Giebels, and L. Foschini for providing details of the H.E.S.S. and the Swift observations of PKS 2155-304. This work is supported by a Grantin-Aid for Scientifi c Research by the Japan Ministry for Education, Culture, Sports, Science and Technology, the Australian Research Council (Grants LE0238884 and DP0345983), and Inter-University Research Program by the Institute for Cosmic Ray Research.

\section{REFERENCES}

1. P. M. Chadwick et al, Astrophys. J. 513, 161 (1999).

2. M. D. Robert et al, Astron. Astrophys. 343, 691 (1999).

3. K. Nishijima et al, Proc. 27th Int. Cosmic Ray Conf.(Hamburg) p. 2626 (2001).

4. K. Nishijima, Publ. Astron. Soc. Australia 19, 26 (2002).

5. T. Nakase et al, Proc. 28th Int. Cosmic Ray Conf.(Tsukuba) p. 2587 (2003).

6. F. Aharonian et al, Astron. Astrophys. 430, 865 (2005).

7. F. Aharonian et al, Astrophys. J. 664, L71 (2007).

8. L. Foschini et al, Astrophys. J. 657, L81 (2007).

9. G. Ghisellini, and F. Tavecchio, MNRAS 386, L28 (2008).

10. M. Kusunose, and F. Takahara, Astrophys. J. 682, 784 (2008).

11. K. Katarzynski et al, accepted in MNRAS (2008).

12. Y. Sakamoto et al, Astrophys. J. 676, 113 (2008).

13. A. Kawachi et al, Astropart. phys. 14, 261 (2001).

14. S. Kabuki et al, Nucl. Instr. Methods:Phy. Res. A 500, 318 (2003).

15. R. Enomoto et al, Astrophys. J. 638, 397 (2006).

16. H. Kubo et al, Proc. 27th Int. Cosmic Ray Conf.(Hamburg) p. 2900 (2001).

17. K. Nishijima et al, Proc. 29th Int. Cosmic Ray Conf.(Pune) 5, 327 (2005)

18. R. Enomoto et al, Astrophys. J. 652, 1268 (2006).

19. W. Benbow et al, Astron. Tel. 867 (2006).

20. F. Aharonian et al, Astrophys. J. 614, 897 (2004).

21. M. Punch et al, Proc. 30th ICRC(Merida) p. \#714 (2007).

22. M. A. Malkov, and L. O'C Drury, Rep. Prog. Phys. 64, 429 (2001).

23. J. R. Primack et al, AIP Conference Proceedings 745, 23 (2005).

24. F. Stecker et al, Astrophys. J. 648, 774 (2006).

25. M. Kino et al, Astrophys. J. 564, 97 (2002). 